\title{
SOLUTIONS OF THE PROBLEMS OF THE 40TH INTERNATIONAL MATHEMATICAL OLYMPIAD
}

\author{
Kevin Hutchinson
}

1. Clearly the vertices of a regular polygon satisfy the conditions in the problem.

If $A$ and $B$ are points in $S$, let $R_{A B}$ denote reflection in the perpendicular bisector of the segment $A B$. Let $G$ be the barycentre of the set $S$ and note that for any two points $A$ and $B$ in $S, R_{A B}(G)=(G)$. Thus $A$ and $B$ are equidistant from $G$. Since this is true for any pair of points of $S$, it follows that the points of $S$ lie on a circle, $\Gamma$, centred at $G$. So the points of $S$ form a convex polygon $A_{1} A_{2} \ldots A_{n}$. Since $R_{A_{1} A_{3}}$ transforms each half-plane bounded by the line $A_{1} A_{3}$ into itself, we must have $R_{A_{1} A_{3}}\left(A_{2}\right)=A_{2}$ (since all other points of $S$ lie on the other halfplane. Thus $A_{1} A_{2}=A_{2} A_{3}$. Similarly, $A_{2} A_{3}=A_{3} A_{4}$ etc, and so the points of $S$ form the vertices of a regular polygon.

2. (Solution communicated by coordinators Marius Radulescu and Cristian Voica.)

$$
\begin{aligned}
\sum_{i<j} x_{i} x_{j}\left(x_{i}^{2}+x_{j}^{2}\right) & \leq\left(\sum x_{i} x_{j}\right)\left(\sum_{i} x_{i}^{2}\right) \\
& =\frac{1}{2}\left(\sum x_{i}^{2}\right)\left(2 \sum x_{i} x_{j}\right) \\
& \leq \frac{1}{2}\left(\frac{\sum x_{i}^{2}+2 \sum x_{i} x_{j}}{2}\right)^{2} \\
& =\frac{1}{8}\left(\sum x_{i}\right)^{4} .
\end{aligned}
$$

Thus $C \leq 1 / 8$. Equality occurs at the first ' $\leq$ ' above if and only if there exists $i \neq j$ such that $x_{k}=0$ for $k \neq i, j$. Suppose this 
is so. The second ' $\leq$ ' is the AM-GM inequality and so equality occurs precisely when $x_{i}^{2}+x_{j}^{2}=2 x_{i} x_{j}$, i.e., when $x_{i}=x_{j}$. Thus $C=1 / 8$ and equality occurs if and only if all but two of the $x_{k}$ 's are zero and the remaining two are equal.

3. First colour the board black and white like a chessboard. Let $f(n)$ be the number we are looking for. Let $f_{w}(n)$ be the minimal number of white squares that must be marked so that any black square has a white neighbour. Similarly define $f_{b}(n)$. Then from considerations of symmetry ( $n$ is even),

$$
f_{b}(n)=f_{w}(n) \text { and } f(n)=f_{b}(n)+f_{w}(n) .
$$

Label the squares $(i, j)$ for $1 \leq i, j \leq n=2 k$ in such a way that $(1,1)$ is white (thus the white squares are those satisfying $i \equiv j$ $(\bmod 2))$. Now mark the (white) squares $(i, j)$ satisfying

$$
\text { (i) } i+j \equiv 2 \bmod 4
$$

and

$$
\text { (ii) } i \text { and } j \text { are } \begin{cases}\text { odd, } & \text { if } i+j \leq n \\ \text { even } & \text { if } i+j>n\end{cases}
$$

(So squares on every second white diagonal are marked, and condition (ii) ensures that the edge squares of each of these diagonals are marked.) Consider an arbitrary black square. If it is in the interior, it has four adjacent white squares and, of these, two satisfy (i). Of these two, one satisfies (ii) and, thus, is marked. On the other hand, if the black square is at an edge, then condition (ii) guarantees that exactly one of the adjacent edge squares is marked. Thus with this marking, every black square is adjacent to exactly one marked square. The total number of marked white squares is $k(k+1) / 2$. Thus $f_{w}(n) \leq k(k+1) / 2$.

On the other hand, no two of the marked white squares have a common adjacent black square, since otherwise two of them would be a distance 2 apart in the 'taxicab' metric $\left(d\left((i, j),\left(i^{\prime}, j^{\prime}\right)\right)=\left|i-i^{\prime}\right|+\left|j-j^{\prime}\right|\right)$, whereas in fact the minimum distance between two marked squares is 4 . Thus we need to mark 
at least $k(k+1) / 2$ black squares in order to cover the marked white squares. Hence $f_{b}(n)=f_{w}(n) \geq k(k+1) / 2$.

To conclude :

$$
f_{w}(n)=f_{b}(n)=\frac{k(k+1)}{2}
$$

and thus,

$$
f(n)=f_{w}(n)+f_{b}(n)=k(k+1)
$$

4. Clearly $n=1$ and $p$ any prime gives a solution.

Suppose now that $n>1$ and let $q$ be the smallest prime dividing $n$; say $n=q s$. Since $(p-1)^{n} \equiv-1 \bmod n^{p-1}$, we have $(p-1)^{q s} \equiv-1 \bmod q \Rightarrow$

$$
(p-1)^{s} \equiv-1 \bmod q
$$

by Fermat's Little Theorem. Now, $(s, q-1)=1$ by choice of $q$. So there exists $a \in \mathbb{N}$ with $a s \equiv 1 \bmod q-1$ and thus, by Fermat's Little Theorem again

$$
p-1 \equiv(p-1)^{a s} \equiv(-1)^{a} \bmod q
$$

But, if $q>2, p-1 \not \equiv 1 \bmod q$ by (1). So $p-1 \equiv-1 \bmod q$, i.e., $q \mid p$, and thus $q=p$, since $p$ is prime.

So, if $n>1$, then $p$ is the smallest prime dividing $n$. Since $n \leq 2 p$, we thus have that $p=2$ and $n=2$ or 4 , or $p$ is odd and $n=p$. Now $p=2$ or 3 and $n=p$ are readily seen to be solutions, while $p=2$ and $n=4$ is not. Suppose finally that $p \geq 5$. Then

$(p-1)^{p}+1=p^{p}-\left(\begin{array}{c}p \\ p-1\end{array}\right) p^{p-1}+\cdots-\left(\begin{array}{c}p \\ 2\end{array}\right) p^{2}+p^{2} \equiv p^{2} \bmod p^{3}$

and thus $p^{p-1}$ does not divide $(p-1)^{p}+1$. So the only solutions are $(p, n)=(2,2),(3,3)$ or $(p, 1)$. 


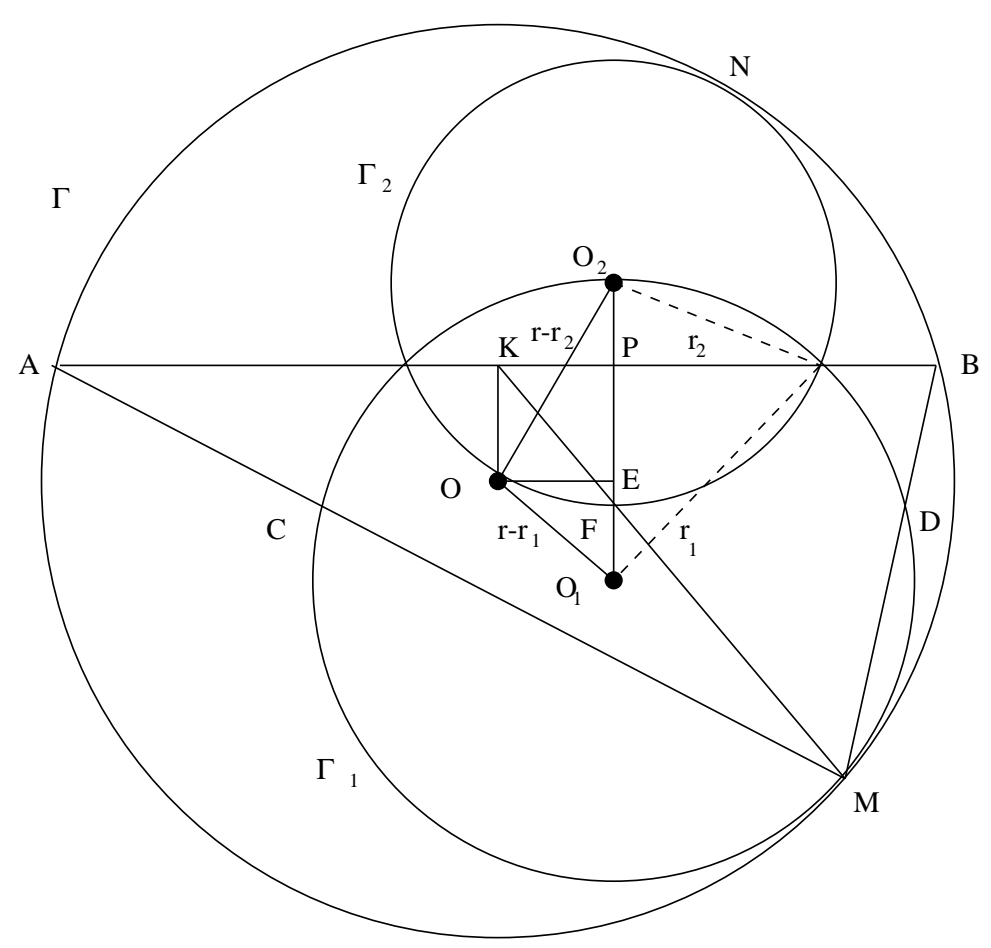

Diagram for Problem 5.

5. (N. Sedrakian) Let $O, O_{1}$ and $O_{2}$ be the centres of $\Gamma, \Gamma_{1}$ and $\Gamma_{2}$ respectively, and $r, r_{1}$ and $r_{2}$ the radii of these circles. Let $K$ on $A B$ and $E$ on $O_{1} O_{2}$ be the feet of the perpendiculars from $O$. Let $F$ be the intersection of $M K$ and $O_{1} O_{2}$. We assume that $r_{1}>r_{2}$. (The other cases, $r_{1}=r_{2}$ and $r_{1}<r_{2}$ can be treated similarly.) 
$\triangle M O_{1} F \sim \triangle M O K\left(\right.$ since $\left.O K \| O_{1} F\right)$. Thus

$$
\begin{aligned}
O_{1} F & =\frac{r_{1}}{r} O K=\frac{r_{1}}{r}\left(O_{1} P-O_{1} E\right) \\
& =\frac{r_{1}}{r}\left(\frac{2 r_{1}^{2}-r_{2}^{2}}{2 r_{1}}-\frac{\left(r-r_{1}\right)^{2}+r_{1}^{2}-\left(r-r_{2}\right)^{2}}{2 r_{1}}\right) \\
& =r_{1}-r_{2} \Rightarrow O_{2} F=r_{2}
\end{aligned}
$$

and thus $F \in \Gamma_{2}$. (Here we use the fact that in a triangle with vertices $A, B, C$ and opposing sides $a, b, c$, the distance from $B$ to the foot of the altitude at $A$ is $\left(a^{2}+c^{2}-b^{2}\right) / 2 a$.)

Since $\triangle M O A$ and $\triangle M O_{1} C$ are isosceles with common base angle $\left\langle C M O_{1}=\langle A M O\right.$, these triangles are similar. The triangles $\triangle M O_{1} D$ and $\triangle M O B$ are likewise similar. Thus

$$
\frac{C M}{A M}=\frac{O_{1} M}{O M}=\frac{r_{1}}{r}=\frac{D M}{B M}=\frac{F M}{K M}
$$

and thus $C D \| A B$ and $F \in C D$. But $A B \perp O_{1} O_{2} \Rightarrow C D \perp O_{2} F$ and thus $C D$ is a tangent to $\Gamma_{2}$.

6. Let $y \in \mathbb{R}$ and $x=f(y)$. Then

$$
\begin{gathered}
f(0)=f(x-f(y))=f(f(y))+f(y)^{2}+f(f(y))-1 \\
\Rightarrow f(f(y))=\frac{c+1}{2}-\frac{f(y)^{2}}{2}=K-\frac{f(y)^{2}}{2} \quad(*)
\end{gathered}
$$

where $c=f(0)$. Note that $f(0) \neq 0$ since, otherwise, taking $y=0$ in the functional equation gives $f(x)=f(x)-1$. Letting $y=0$ in the functional equation, we get:

$f(x-c)=f(c)+c x+f(x)-1 \Rightarrow f(x-c)-f(x)=f(c)-1+c x$ is a non-constant linear function of $x$.

Thus, given $z \in \mathbb{R}$, there exists $x \in \mathbb{R}$ with $z=f(x-c)-f(x)=$ $f(w)-f(x)$, say. Hence for $z \in \mathbb{R}$, 


$$
\begin{aligned}
f(z) & =f(f(w)-f(x)) \\
& =f(f(x))+f(w) f(x)+f(f(w))-1 \\
& \left.=K-\frac{f(x)^{2}}{2}+f(w) f(x)+K-\frac{f(w)^{2}}{2}-1 \quad \text { (using }(*)\right) \\
& =2 K-1-\frac{(f(w)-f(x))^{2}}{2}=2 K-1-\frac{z^{2}}{2}
\end{aligned}
$$

Taking $z=f(y)$ for any $y$ and using $(*)$, we get $2 K-1=K$ so that $K=1$ (and $c=f(0)=1$ ) and thus

$$
f(z)=1-\frac{z^{2}}{2}
$$

for all $z \in \mathbb{R}$. It is easily verified that this function does satisfy the functional equation.

\section{Kevin Hutchinson}

Department of Mathematics

University College Dublin

Dublin 